\title{
Situations didactiques et enseignement de la grammaire: quelques aspects topogénétiques et chronogénétiques
}

\section{Marie-Claude Boivin}

Cet article se fonde sur l'analyse d'interactions didactiques portant sur la phrase de base et la subordonnée relative en classe de français, et présente deux propositions relatives au déroulement des situations didactiques. Il est soutenu qu'il existe des conditions topogénétiques à l'avancement du savoir en classe (chronogenèse), plus spécifiquement que l'anticipation des hypothèses alternatives des élèves et la clarté des représentations de l'enseignant relativement au savoir à enseigner sont cruciales. Deux caractéristiques d'un élève chronogène sont proposées et illustrées dans une leçon de grammaire: la capacité de conciliation et l'utilisation des manipulations syntaxiques. Le rôle des connaissances grammaticales implicites (compétence linguistique) des élèves dans l'enseignement grammatical est discuté.

\section{Introduction}

À l'instar d'autres didactiques, la didactique du français s'intéresse de plus en plus à l'observation directe des interactions en classe de français (Canelas-Trevisi, 1998; Canelas-Trevisi, Moro, Schneuwly \& Thévenaz, 1999; Schubauer-Leoni \& Dolz, 2004; Wirthner \& Schneuwly, 2004). Dans la foulée de ces travaux, nous présentons ici les résultats de l'analyse d'interactions didactiques portant sur l'enseignement/apprentissage de la subordonnée relative dans une classe de français de huitième année en Suisse romande ${ }^{1}$. Les extraits retenus concernent l'analyse d'une subordonnée relative en dont (la femme dont la beauté égalait la santé): les élèves devaient reconstruire la phrase de base (ci après, $\mathrm{P}$ de base) correspondante. Nous avons constaté un écart entre la P de base selon la théorie de référence (la beauté de la femme égalait la santé de la femme) et la phrase institutionnalisée par l'enseignant (la beauté égalait la santé de la femme). Cet écart a contribué à créer un blocage de la situation didactique et le travail de reconstruction de la $\mathrm{P}$ de base a suscité chez les élèves des interventions nombreuses et variées, visant à débloquer la situation, à la faire avancer. L'analyse fine de cette situation didactique éclaire à notre avis certains aspects du fonctionnement du 
système didactique et contribue à une meilleure compréhension de ce système (Brousseau, 1998).

Cet article convoque deux paradigmes théoriques qui ont peu souvent l'occasion de cohabiter. Il s'agit d'une part des théories de la transposition didactique (Chevallard, 1985/1991) et des situations didactiques (Brousseau, 1998) et, d'autre part, de la théorie de la grammaire générative (Chomsky, 1981, 1986, 1995).

Chevallard (1985/91) distingue transposition didactique externe et interne. La première concerne le passage des savoirs savants aux savoirs à enseigner ${ }^{2}$; la seconde le passage des savoirs à enseigner aux savoirs effectivement enseignés en classe. L'analyse de la transposition didactique interne fait notamment appel aux concepts de chronogenèse et de topogénèse; nous présentons ici deux propositions didactiques liées à ces concepts et plus précisément au lien qui existe entre eux. Nous soutiendrons dans un premier temps que la conduite de la chronogenèse est soumise à des conditions topogénétiques, plus spécifiquement que le rapport au savoir de l'enseignant détermine en partie sa conduite de la chronogenèse. Ces conditions topogénétiques seront illustrées: elles incluent l'anticipation des hypothèses alternatives des élèves et la clarté des représentations de l'enseignant relativement au savoir à enseigner. La seconde proposition vise à énoncer certaines caractéristiques de l'élève chronogène (Leutenegger \& Schubaeur-Leoni, 2002; Sensevy, 1998), qui seront également illustrées spécifiquement pour l'enseignement grammatical: elles incluent la capacité de conciliation et l'utilisation des manipulations syntaxiques.

Les aspects méthodologiques essentiels sont présentés dans la deuxième partie. L'objet d'enseignement est traité dans la troisième partie: les connaissances grammaticales transposées liées à la subordonnée relative, la tâche des élèves et ses difficultés y sont présentées. Viennent ensuite les éléments clés de l'analyse de la grammaire générative pour la phrase travaillée en classe, incluant certaines connaissances implicites chez le locuteur relativement à la phrase en question. En nous fondant sur cette analyse a priori de l'objet, nous complétons le volet méthodologique en motivant dans la quatrième partie le choix des extraits retenus. Nos propositions relativement aux conditions topogénétiques déterminant la conduite de la chronogenèse en classe et relativement aux caractéristiques des élèves chronogènes sont soutenues dans les cinquième et sixième partie respectivement.

\section{Aspects méthodolgiques}

Les données proviennent d'un corpus de leçons filmées dans des classes de français de huitième année en Suisse romande ${ }^{3}$. Ce corpus comprend deux ensembles de leçons portant respectivement sur la subordonnée relative et sur le texte d'opinion. Nous avons retenu pour fins d'analyse une séquence de six minutes, faisant partie de la deuxième leçon d'une série de quatre portant sur la subor- 
donnée relative, et nous en avons effectué la transcription. L'analyse a priori de l'objet enseigné et de ses propriétés linguistiques nous a permis d'identifier dans cette séquence des événements remarquables (Schubauer-Leoni \& Leutenegger, 2002). Les critères utilisés pour le choix des extraits sont présentés dans la quatrième partie.

Nous adoptons une approche descriptive, et non prescriptive. L'observation et la description fine d'une situation didactique particulière permet ici de contribuer à la théorie de la transposition didactique, plus particulièrement à celle de la transposition didactique interne, en affinant le concept de chronogenèse et en établissant un lien entre chronogenèse et topogénèse. La situation sous étude est bloquée, et nous n'offrons pas de solution (prescriptive) pour la débloquer: nous observons simplement ses caractéristiques et en tirons des conclusions pour la théorie.

\section{L'objet d'enseignement: difficultés et analyse a priori de l'objet enseigné}

Cette partie contient les informations essentielles pour comprendre la tâche des élèves, les difficultés qu'elle présente et les connaissances déjà présentes chez eux relativement à l'objet. La première section situe de façon générale les savoirs en cause en traitant de la transposition didactique externe. La deuxième section porte sur la structure de la subordonnée relative en grammaire pédagogique moderne et explicite la tâche des élèves. La troisième section identifie précisément les difficultés liées à la phrase retenue pour cette recherche; la quatrième section présente les caractéristiques syntaxiques de cette phrase (phrase à vides parasites) selon la grammaire générative et enfin la cinquième section fait état des connaissances grammaticales implicites des élèves sur la subordonnée relative.

\section{Une note sur la transposition didactique externe}

Le travail en classe a été effectué par les élèves dans le cadre de la grammaire dite «nouvelle», ou grammaire moderne. La grammaire «nouvelle» a déjà plus de trente ans; elle s'inspire des travaux de linguistique moderne, entre autres ceux de la grammaire générative. Elle vise à décrire, à des fins d'apprentissage scolaire, les grandes régularités syntaxiques de la langue, délaissant les descriptions de la grammaire traditionnelle, fondées sur la sémantique et plus ou moins adéquates sur le plan descriptif (Chartrand, 1996; Nadeau \& Fisher, 2006). Une telle grammaire scolaire ne peut utiliser directement les résultats de travaux de linguistique théorique sans les adapter, les transposer, les didactiser. Le travail de transposition didactique externe inclut une réflexion sur le choix des contenus à retenir des savoirs savants et leur reconfiguration (Vargas, 2004) à des fins d'enseignement et d'apprentissage (voir notamment Gobbe, 1980). Ce travail doit tenir compte des capacités des élèves, de la pertinence des contenus retenus 
et de la cohérence du système présenté à l'élève (Schneuwly, 2005). Pour faciliter l'exposé, nous présenterons dans le corps du texte les descriptions transposées, i.e. celles qui se retrouvent dans les documents pédagogiques (grammaires, manuels, cahiers, etc.) et avec lesquelles les élèves travaillent. Les analyses linguistiques seront brièvement présentées dans les notes.

\section{La subordonnée relative et la reconstruction de La $P$ de base en grammaire nouvelle}

\section{La structure de la subordonnée relative}

Dans les documents pédagogiques (grammaires, manuels, etc.), la subordonnée relative est analysée comme une phrase enchâssée comme complément du nom dans un syntagme nominal $(\mathrm{SN})^{4}$, lui-même partie d'une autre phrase (voir, entre plusieurs autres Genevay, 1994; Riegel, Pellat \& Rioul, 1994/2004). La phrase dans laquelle se retrouve la subordonnée relative s'analyse donc comme impliquant deux phrases d'origine. Ainsi, la phrase (1) est formée des phrases P1 et $\mathrm{P} 2$, données en $(2 \mathrm{a})$ et $(2 \mathrm{~b})$.

(1) Le phénomène que Jean a expliqué est passionnant.

(2) a. Le phénomène est passionnant.

b. Jean a expliqué le phénomène.

La subordonnée relative que Jean a expliqué est dérivée comme suit ${ }^{5}$. Le SN le phénomène de $\mathrm{P} 2$, contenant le terme commun aux deux phrases (phénomène), est remplacé par le pronom relatif que, qui se déplace en tête de phrase, comme illustré en (3) et (4).

(3) Jean a expliqué < que >

(Remplacement de le phénomène par le pronom relatif que)

(4) que Jean a expliqué <

(Déplacement de que en tête de phrase)

Le résultat en (4) est la subordonnée relative, qui peut être enchâssée dans le SN le phénomène de P1, comme illustré en (5a) et (5b).

a. [Le phénomène] est passionnant.

b. [Le phénomène que Jean a expliqué] est passionnant. (Enchâssement)

Les exemples (6) à (8) illustrent la construction de la subordonnée relative avec le pronom relatif dont. Lorsque dont remplace un syntagme prépositionnel $(\mathrm{SP})^{6}$, ce syntagme a pour noyau la préposition de; dans ce cas précis il se substitue au $\mathrm{SP}$ de ce livre, complément du nom conclusion. 
(6) a. Julie a acheté [un livre]. $\quad$ P 1

b. La conclusion $<$ de ce livre $>$ est étonnante. P2

(7) a. la conclusion <dont $>$ est étonnante (remplacement)

b. dont la conclusion $<\ldots$ _ est étonnante_ (déplacement)

(8) Julie a acheté [un livre dont la conclusion est étonnante].

(enchâssement)

\section{La tâche des élèves: reconstruire la P de base}

Dans la première des quatre leçons, les élèves ont eu à reconstruire les deux $\mathrm{P}$ de base à la source de phrases contenant une relative. Ces dernières présentant toutefois de nombreuses difficultés d'interprétation, l'enseignant propose au début de la deuxième leçon un exercice semblable, portant sur huit phrases extraites d'un conte lu auparavant. Ce choix a pour but d'assurer une certaine familiarité des élèves avec le contenu et ainsi de diminuer les difficultés d'interprétation éprouvées lors de la première leçon.

Les phrases sur lesquelles les élèves travaillent dans cette deuxième leçon contiennent des subordonnées relatives soulignées par l'enseignant. La tâche des élèves consiste à reconstruire, à partir d'une phrase complexe, la $\mathrm{P}$ de base associée à la relative. Ils doivent identifier le groupe syntaxique remplacé par le pronom relatif, placer ce groupe dans la phrase et indiquer la transformation effectuée par un cadre autour du groupe et une flèche jusqu'au pronom. Voici un exemple de la tâche des élèves:

(9) Les enfants que j’ai rencontrés m’ont semblé joyeux.

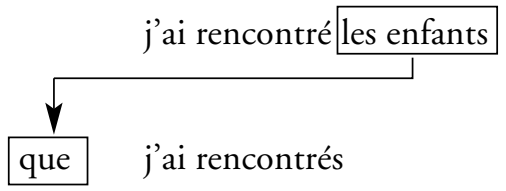

Notons au passage un aspect de l'intérêt didactique d'une telle analyse: le lien entre le SN les enfants et le pronom relatif que est explicite; les deux correspondent à la même fonction grammaticale (complément direct, CD). À partir de l'exemple (9), l'élève est à même de comprendre que le pronom que complément direct commande l'accord du participe rencontrés: le CD s'est déplacé devant le participe.

La difficulté de reconstruction de La $P$ de base associée

à la relative

La phrase sur laquelle les élèves travaillent est donnée en (10).

(10) La sage-femme prédit que cette femme, dont la beauté égalait la santé, accoucherait à coup sûr d'un ravissant petit garçon. 
Les élèves travaillent uniquement sur la subordonnée relative dont la beauté égalait la santé et doivent reconstruire la $\mathrm{P}$ de base qui y est associée. Cette dernière, donnée en (11), est difficile à reconstruire: en effet, le pronom relatif dont semble avoir été extrait de deux sites (à la fois du sujet et du complément).

(11) La beauté de cette femme égalait la santé de cette femme.

La première difficulté provient de ce que la reconstruction de la $\mathrm{P}$ de base semble exiger la violation d'un principe rarement, sinon jamais, explicitement formulé dans le travail grammatical pédagogique. En effet, un principe, que nous appellerons principe d'unicité, contraint la transformation de substitution (remplacement) en grammaire générative: un même élément ne peut remplacer qu'un seul groupe de mots dans une phrase. Or, en (11), il semble que dont remplace les deux occurrences du groupe de cette femme, en violation du principe d'unicité (la structure de la phrase est donnée plus loin en 3.4). Bien que le bon fonctionnement de la manipulation syntaxique de substitution repose sur le respect du principe d'unicité, les transpositions didactiques disponibles ne le mentionnent pas explicitement. En effet, lorsqu'on demande aux élèves de remplacer un groupe par un autre élément, il est sous-entendu qu'il ne faut remplacer qu'un seul groupe à la fois. Ici, l'emprunt de la transformation de substitution dans la transposition didactique implique également l'emprunt de ce principe, sans lequel la transformation ne peut fonctionner adéquatement. Cet état de fait montre les limites et les exigences de la transposition didactique relativement au choix des contenus à enseigner: certains emprunts effectués aux savoirs savants impliquent l'importation dans les savoirs à enseigner de contraintes, qui, si elles sont fermement ancrées dans les savoirs théoriques de référence, auraient avantage à être explicitées dans la transposition.

La seconde difficulté est liée au fait que la P de base reconstruite ne peut, dans des conditions normales de discours, être énoncée telle quelle. En effet, une même expression référentielle (ici cette femme) ne peut en général pas être utilisée deux fois dans la même phrase 7 . Si la phrase était énoncée, le déterminant possessif anaphorique (sa) devrait être utilisé, comme il est illustré en (12). Le caractère «non énonçable» de la $\mathrm{P}$ de base contribue à rendre sa reconstruction difficile.

(12) La beauté de la femme égalait sa santé.

Compte tenu des difficultés inhérentes à la subordonnée relative donnée en (10), il aurait sans doute été préférable de ne pas la travailler avec des élèves. Il sera montré dans la quatrième partie de l'article que l'enseignant n'a pas anticipé les difficultés associées à cette phrase: ce n'est pas intentionnellement qu'elle a été présentée aux élèves. En tout état de cause, dans notre perspective descriptive et théorique, exempte de prescriptivisme, les interventions portant sur cette phrase méritent l'attention du didacticien: elles sont révélatrices du fonctionnement du système didactique. 


\section{Éléments fondamentaux de L'analyse syntaxique des con- structions à vides parasites}

Revenons à la première difficulté: le pronom relatif dont semble avoir deux origines dans la P de base, comme l'illustrent (13) et (14).

(13) La beauté de cette fermme égalait la santé de cette femme.

(14) La femme dont [la beauté __ ] égalait [la santé

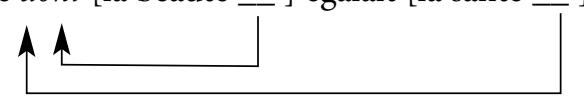

Le phénomène illustré en (14), connu sous le nom de constructions à «vides parasites» (parasitic gaps, cf. Chomsky, 1981), a reçu beaucoup d'attention en grammaire générative.

Les constructions à vides parasites se caractérisent par la présence de deux positions vides dans la structure, correspondanct sémantiquement à un seul élément dans la phrase (ici, dont). La structure de la relative dont la beauté égalait la santé a, selon les analyses de la grammaire générative, les caractéristiques données en $(15)^{8}$ :

(15) La femme1 dont $t_{1}$ [la beauté__ ] égalait [la santé $\left.\varnothing_{1}\right]$.

L'observation cruciale est la suivante: l'extraction du dont de la position sujet permet d'avoir un complément du nom vide $\left(\varnothing_{1}\right)$ ayant la même interprétation (ceci est noté par le partage de l'indice 1 ). Ce phénomène est appelé vide parasite parce que la position vide dans le complément n'est possible que s'il y a une position vide dans le sujet. Le «vide» dans le complément est donc dépendant (parasite) du «vide» dans le sujet.

Le phénomène des vides parasites se retrouve régulièrement en français (Tellier, 1991; Tellier, 2001; Tellier \& Valois, 2006); la phrase sur laquelle les élèves travaillent ne constitue donc pas un cas isolé9.

\section{Connaissances implicites du locuteur sur la subordonnée relative}

Dans le but de faciliter la compréhension des interventions des élèves, nous présentons dans cette section certaines connaissances que possède tout locuteur du français, selon la grammaire générative, sur la subordonnée relative et sur les constructions à vides parasites.

La grammaire générative a pour but d'élaborer un modèle décrivant la compétence linguistique du locuteur natif, sa grammaire interne (Chomsky, 1957, 1965; Pollock, 1997). Il s'agit d'un modèle formel qui décrit les connaissances linguistiques présentes chez le locuteur. Ces connaissances lui permettent entre autres de déterminer si une phrase fait partie ou non de sa langue, elles se mani- 
festent donc notamment par le jugement de grammaticalité. Souvent, elles demeurent implicites, c'est-à-dire que le locuteur ne pourrait expliquer les raisons pour lesquelles il juge telle phrase grammaticale ou agrammaticale (DeKeyser, 2003; Fetzer, 2004; Kak, 1992). Cet aspect est bien résumé par Kak (1992):

Every native speaker of a language evidently knows what is and what isn't a sentence of his language (...) but even a linguistically sophisticated one may be hard put in a given case to say explicitely what underlying principles are involved (p. 5-6).

La grammaire générative ne propose pas un modèle de production langagière, qui viserait à expliquer comment le locuteur construit sa phrase avant de la prononcer ou de l'écrire, mais bien un modèle de la connaissance qu'a le locuteur des propriétés de sa langue.

Voici deux connaissances (implicites) cruciales que possède un locuteur du français sur la subordonnée relative. D'abord, la position d'origine du pronom relatif ${ }^{10}$ n'est pas disponible pour y placer un autre élément, comme il est illustré en (16).

(16) a. Le problème que Jean a expliqué était complexe.

b. *Le problème que Jean a expliqué l'exercice était complexe.

Si on essaie d'insérer un SN comme l'exercice dans la position de complément du verbe a expliqué, comme en (16b), on obtient une phrase agrammaticale (L'agrammaticalité est notée par l'astérisque). Cette position correspondant à la position d'origine du pronom relatif (voir exemple 3, plus haut), l'analyse prédit correctement qu'on ne peut y retrouver un autre élément.

La seconde connaissance, qui découle en partie de la première, est que la position d'origine du pronom relatif est associée à ce dernier: non seulement elle n'est pas disponible, mais elle est occupée par un élément phonologiquement nul et issu du déplacement du pronom. Cette connaissance permet de reconstruire la $P$ de base.

Pour la subordonnée relative à l'étude, dont la représentation est donnée en (15), la grammaire générative prédit que le locuteur a entre autres les connaissances implicites données en (17).

(17) Compétence linguistique du locuteur natif du français pour la relative à l'étude

a. Il y a deux positions de complément du nom.

b. Ces positions sont occupées par un élément phonologiquement nul et sémantiquement identique à dont.

Comme on le verra lors de l'examen des interactions didactiques, ces connaissances se sont manifestées à travers les interventions des élèves. 


\section{Le choix des interactions didactiques}

L'analyse approfondie de l'objet selon le savoir théorique de référence a orienté notre sélection des interactions didactiques susceptibles de révéler le fonctionnement du système didactique. Nous présentons donc les caractéristiques générales de la situation didactique retenue et son intérêt, puis les critères formels utilisés pour le choix des interactions.

\section{Intérêt de la situation didactique}

Compte tenu des difficultés déjà discutées, il n'est pas étonnant de constater que la reconstruction de la $\mathrm{P}$ de base a été problématique, pour les élèves comme pour l'enseignant. Nous ne prétendons pas que des phrases aussi complexes devraient faire l'objet d'un enseignement au secondaire; il serait idéal que l'enseignant soit en mesure de les exclure.

\section{Le savoir à enseigner}

De façon générale, dans le contexte, le savoir à enseigner vise à développer chez les élèves la capacité de faire le lien entre une phrase transformée (ici une subordonnée relative) et la $\mathrm{P}$ de base correspondante (voir exemple 1, plus haut). Notons tout d'abord que l'écart relevé et discuté plus bas est exceptionnel dans la leçon: le savoir à enseigner ne pose en général aucun problème. Ainsi, pour la plupart des exercices, les élèves, guidés par l'enseignant, utilisent leur savoir de façon pertinente et atteignent les résultats attendus.

\section{Une différence entre la phrase conforme au savoir théorique et la phrase institutionnalisée}

La difficulté posée par la relative de la phrase (10) a toutefois créé une situation où il existe une différence entre la $\mathrm{P}$ de base conforme au savoir théorique de référence et la $\mathrm{P}$ de base effectivement institutionnalisée par l'enseignant, c'est-àdire présentée à l'ensemble de la classe comme ayant le statut de savoir (Brousseau, 1998). En effet, l'enseignant n'a pas reconstruit la $\mathrm{P}$ de base conforme au savoir de théorique de référence, soit (18) - dorénavant notée $\mathrm{P}_{\mathrm{T}}$ (phrase conforme au savoir théorique) - mais plutôt la phrase (19) - dorénavant notée $\mathrm{P}_{\mathrm{I}}$ (phrase institutionnalisée) - où le SP de cette femme n'apparaitt qu'une seule fois.

(18) $\mathrm{P}_{\mathrm{T}}$ La beauté de cette femme égalait la santé de cette femme

(19) $\mathrm{P}_{\mathrm{I}}$ La beauté égalait la santé de cette femme ${ }^{11}$.

La $\mathrm{P}$ de base telle que reconstruite par l'enseignant $\left(\mathrm{P}_{\mathrm{I}}\right)$ differe de la $\mathrm{P}$ de base conforme $\left(\mathrm{P}_{\mathrm{T}}\right)$ en deux points: elle ne comprend qu'un SP (de cette femme) et non deux; le SP est donné comme complément de santé et non comme complément de beauté. Les interventions des élèves qui ont suivi l'institutionnalisation de $\mathrm{P}_{\mathrm{I}}$ sont particulièrement intéressantes parce qu'elles pointent vers $\mathrm{P}_{\mathrm{T}}$. 


\section{Critères pour le choix des interactions: Les événements} remarquables

Nos hypothèses seront soutenues par cinq extraits de la leçon qui contiennent des événements remarquables, plus précisément des interventions remarquables des élèves, pointant vers la différence entre $\mathrm{P}_{\mathrm{I}}$ et $\mathrm{P}_{\mathrm{T}}$. Schubauer-Leoni \& Leutenegger (2002, p. 246) définissent les événements remarquables comme des «moments cruciaux et emblématiques de la séance, relativement au questionnement de recherche». Ici, les événements remarquables sont bien des moments cruciaux, mais ils ne sont pas emblématiques, ou prototypiques, de la séance. En effet, la séance d'enseignement dans son ensemble ne se caractérise pas par la présence systématique d'écarts entre des $\mathrm{P}_{\mathrm{T}}$ et des $\mathrm{P}_{\mathrm{I}}$, bien au contraire. Ce qui caractérise un événement remarquable dans cette séquence d'enseignement, c'est justement son caractère atypique (et non emblématique) par rapport à l'ensemble. Les interventions de certains élèves sont remarquables parce que (1) il existe une différence entre savoir conforme au savoir théorique et savoir institutionnalisé et (2) l'intervention vise cette différence.

Nous avons retenu toute intervention pouvant être interprétée comme visant un aspect de la $\mathrm{P}_{\mathrm{T}}$. Les critères sont donc que l'intervention porte sur:

a) la position du SP (de cette femme) dans la phrase (à la fin, au début, pas là, etc.); ou

b) le lien entre le SP et le noyau nominal beauté.

La discussion ne constitue nullement une critique du travail de l'enseignant; le blocage de la situation didactique observé dans le travail sur la relative agit comme un révélateur du mode de fonctionnement du système didactique, et c'est sur ce fonctionnement que nous nous attarderons dans les parties qui suivent.

\section{Chronogenèse et topogenèse dans l'enseignement grammatical}

Dans cette partie nous soutiendrons l'hypothèse qu'il existe des conditions topogénétiques déterminant la conduite de la chronogenèse: cette dernière dépend de la position de l'enseignant par rapport au savoir, de son rapport au savoir, bref, d'aspects topogénétiques.

\section{Le concept de chronogenèse}

Le concept de chronogenèse a été proposé par Chevallard (1985/91) et peut être défini comme l'avancement du savoir dans la classe en fonction du temps, «la production des savoirs au fil de la temporalité didactique» (Schubauer-Leoni \& Leutenegger, 2002). Selon Chevallard (1985/91, pp. 71-74), la chronogenèse est 
«conduite» par l'enseignant ${ }^{12}$, qui décide notamment du moment de l'introduction de nouveaux objets de savoir en classe. La conduite de la chronogenèse est rendue possible grâce à la position temporelle de l'enseignant par rapport au savoir. Comme le souligne Chevallard (1985/91, p. 71): «L'enseignant est donc celui qui sait avant les autres, qui sait déjà, qui sait "plus». Et cela lui permet de conduire la chronogénèse du savoin» ${ }^{13}$.

\section{Des conditions topogénétiques déterminant la conduite de}

\section{la chronogenèse}

Nous proposons que la conduite de la chronogenèse, en plus de dépendre de la position temporelle de l'enseignant, dépend également de sa position topogénétique, i.e sa position par rapport au savoir. La position topogénétique de l'enseignant se caractérise par un rapport différent au savoir à enseigner, ce que Chevallard (1985/91, p. 75) appelle son «savoir autrement». Il existe donc à notre avis des conditions topogénétiques à la conduite de la chronogenèse, dont les deux conditions énoncées ci-dessous et subséquemment traitées tour à tour.

(20) a. l'anticipation des hypothèses des élèves;

b. la clarification des contraintes implicites perçues par l'enseignant relativement au savoir à enseigner.

\section{L'anticipation des hypothèses alternatives des élèves}

L'extrait 1 (plus bas) est selon nous révélateur d'une condition topogénétique essentielle à la conduite de la chronogenèse, qui vient de plus préciser une modalité du "savoir autrement» de l'enseignant, soit l'anticipation et la prise en compte des hypothèses alternatives des élèves. Soulignons le fonctionnement de notre argumentation. Nous n'affirmons pas que, dans la situation sous étude, l'enseignant en question aurait dû avoir un rapport différent au savoir: un enseignant n'a pas à maitriser toutes les subtilités des savoirs théoriques de référence. Ce que montre a contrario cette portion de la situation didactique, avec un cas extrême, c'est tout simplement qu'un rapport au savoir différent permet d'anticiper les hypothèses alternatives des élèves, et donc participe à la chronogenèse, ce qui devrait être vrai de façon plus générale, au-delà de la situation spécifique en cause.

Au moment où se déroule l'extrait 1 , les élèves ont travaillé individuellement sur la relative dont la beauté égalait la santé. Ils éprouvent des difficultés, et l'enseignant ouvre une discussion générale. Dans les extraits, $\mathrm{E}$ désigne un élève et $\mathrm{P}$ désigne l'enseignant; le temps indiqué renvoie à la chronologie du cours (en minutes et secondes). 


\section{Extrait 1}

\begin{tabular}{|c|c|c|}
\hline Temps & & verbatim \\
\hline & $\mathrm{P}$ & VOUS ETES TOUS CROCHÉS ${ }^{13}$ SUR CELLE-LÀ \\
\hline $14: 58$ & E1 & Oui c'est bon j'sais monsieur $\uparrow$ la beauté égalait $\uparrow / /$ la beauté de cette femme $\uparrow$ \\
\hline & $\mathrm{P}$ & Oui, alors c'est presque. La beauté / égalait / la santé / de cette femme. BRA:VO (Rire) \\
\hline $15: 01$ & E2 & Mais on peut pas dire, heu/ La beauté de cette femme égalait la santé? \\
\hline
\end{tabular}

Une première intervention interprétée en fonction de $P_{I}$

Un élève (E1) propose "la beauté égalait $\uparrow . .$. la beauté de cette femme^», une intervention pouvant être interprétée de deux façons. On peut penser que l'élève s'est trompé et a répété beauté au lieu de dire santé comme noyau du SN complément du verbe égalait (il aurait donc voulu dire La beauté égalait la santé de cette femme). Il est également possible que l'élève ait voulu, après avoir prononcé le verbe, revenir sur le sujet et le compléter. En effet, l'intonation est ascendante après égalait (ceci est noté par $\uparrow$ ), et l'élève fait une pause entre le verbe et le $S N$ qui suit, la beauté de cette ferme. Ce dernier se termine également avec une intonation ascendante, ce qui porte à croire que l'élève n'était pas en train d'énoncer le SN complément du verbe mais bien de répéter le $S N$ sujet. L'enseignant a choisi la première interprétation. Il est probable que sa propre reconstruction de la phrase $\left(\mathrm{P}_{\mathrm{I}}\right)$ l'a fait pencher vers la première interprétation et l'a empêché de considérer la seconde.

Cet extrait révèle que l'instanciation du savoir à enseigner tel que compris par l'enseignant peut constituer un obstacle à sa compréhension des interventions des élèves, l'empêchant de considérer les hypothèses alternatives que les élèves se forment relativement à l'objet de savoir.

Nous proposons donc une certaine précision des conditions nécessaires à la conduite de la chronogenèse du savoir. Chevallard pose en conditions nécessaires au déploiement de la chronogenèse certains aspects du savoir de l'enseignant. Ainsi l'enseignant doit savoir avant, et doit savoir plus, c'est l'aspect "quantitatif» de la différence entre ses savoirs et ceux des élèves. Cependant, Chevallard ne semble pas considérer la position topogénétique de l'enseignant, qui présente un aspect qualitatif, comme une condition déterminant la conduite de la chronogenèse. Il lie plutôt la topogenèse à la position synchronique de l'enseignant: «Car non seulement l'enseignant, supposé savoir et supposé anticiper, doit montrer qu'il peut conduire la chronogénèse didactique, affirmant ainsi son pouvoir dans la diachronie, mais encore il va, en synchronie, affirmer le caractère singulier de sa place propre dans la construction du savoir: non content de savoir plus et de programmer le futur, il sait autrement ${ }^{15}$."

Ầ notre avis, la conduite de la chronogenèse exige la prise en compte par l'enseignant des hypothèses alternatives que pourraient se former les élèves au fur et à mesure que le savoir avance, que se déploie la chronogenèse. Il s'agit d'une condition topogénétique déterminant le déroulement de la chronogenèse. On peut 
la décrire ainsi: l'enseignant a pour rôle de créer les conditions dans les quelles les élèves pourront acquérir une connaissance $\mathrm{x}_{\mathrm{n}}$. Le passage d'un état de connaissance $\mathrm{x}_{\mathrm{i}}$ à un état de connaissance $\mathrm{x}_{\mathrm{n}}$ peut se faire en plusieurs étapes, au fil desquelles certaines bifurcations risquent de se présenter. Les élèves peuvent, au fur et à mesure que leur compréhension avance, emprunter ces bifurcations, en se formant des hypothèses alternatives incorrectes. La prise en considération de ces hypothèses - notamment en les anticipant ${ }^{16}$ - et leur réfutation par l'enseignant nous semble donc un élément essentiel dans la conduite de la chronogenèse. L'anticipation des hypothèses alternatives et leur prise en compte ne peut être assurée que par un rapport au savoir différent du côté de l'enseignant: non seulement il est capable d'utiliser le savoir pour arriver à une application juste, mais il est capable de prévoir les points de bifurcations possibles.

\section{L'expression directe d'une hypothèse alternative par une élève}

À la fin de l'extrait 1, une élève (E2) pose la question: «On peut pas dire, heu... la beauté de cette femme égalait la santé ?». La possibilité de reconstruire la $\mathrm{P}$ de base en replaçant le SP de cette femme dans la position de complément de beauté, directement soulevée, va à l'encontre de $\mathrm{P}_{\mathrm{I}}{ }^{17}$. Cette deuxième intervention porte sur la possibilité que le SP de cette femme soit complément du nom beauté. Encore une fois, cette hypothèse ne semble pas avoir été envisagée par l'enseignant; la condition topogénétique permettant la conduite de la chronogenèse n'est pas remplie, et la situation didactique piétine.

En somme, l'anticipation des hypothèses alternatives des élèves fait à notre avis partie du «savoir autrement» de l'enseignant et constitue une condition topogénétique à la conduite de la chronogenèse, cette conduite exigeant l'anticipation des points de bifurcations possibles dans le raisonnement des élèves.

\section{La clarification des contraintes implicites relativement au savoir à enseigner}

L'extrait 2 commence avec la dernière phrase de l'extrait 1 et présente les interactions entre l'enseignant et l'élève E2 au sujet de sa proposition de P de base.

\section{Extrait 2}

\begin{tabular}{|l|l|l|}
\hline Temps & E/P & Verbatim \\
\hline $15: 01$ & E2 & Mais on peut pas dire, heu/ La beauté de cette femme égalait la santé ? \\
\hline $\begin{array}{l}15: 07 \\
15: 11\end{array}$ & $\mathrm{P}$ & Heu/// (5 “) \\
\hline $15: 17$ & $\mathrm{E} 2$ & $\begin{array}{l}\text { Non, parce que t'es obligé de rajouter un mot, alors, t'es obligé de dire La beauté } \\
\text { de cette femme égalait SA santé à ce moment-là }\end{array}$ \\
\hline $15: 36$ & & $\begin{array}{l}\text { Non ça va pas// non non ceste fermme égalait la santé (16”) } \\
\text { élève) oui } \uparrow\end{array}$ \\
\hline
\end{tabular}


L'hypothèse de l'élève est rejetée parce qu'elle impliquerait de rajouter un mot à la phrase: "La beauté de cette femme égalait SA santé.». Cette réponse de l'enseignant permet de constater le caractère actif chez lui du principe d'unicité, qui contraint la substitution, dans le contexte de la reconstruction de la P de base: il est pour lui impossible de «rajouter un mot à la phrase». Cette représentation de l'enseignant est en général correcte, mais dans la situation didactique en cause, sa rigidité constitue un frein à la conduite de la chronogenèse.

La question de l'élève aurait pu être l'occasion d'une remise en question par l'enseignant du savoir institutionnalisé (sa $\mathrm{P}$ de base $\mathrm{P}_{\mathrm{I}}$ ). La question met bien en lumière le fait que le SP de la femme «va avec» la beauté, sur le plan du sens. En effet, le SN la beauté ne peut être interprété que comme signifiant «la beauté de la femme», il ne s'agit pas de la beauté en général, ni de la beauté de quelqu'un d'autre. Il aurait donc été possible, à ce stade, de considérer la possibilité de reconstruire de cette femme comme complément du nom beauté.

L'apparition du mot sa dans le SN complément a agi comme un frein, car elle contrevient au principe d'unicité. L'intuition grammaticale révélée par la question (de la femme "va avec» la beauté, sinon exclusivement du moins autant qu'avec la santé) n'a pas été prise en compte, ni l'identité de sens entre la relative dont la beauté égalait la santé et La beauté de cette femme égalait sa santé. Il est possible également que la remise en question du savoir à enseigner portée par cette question ait été trop perturbante pour l'enseignant, et que la prise en compte de cette nouvelle donnée lui aurait causé un réajustement trop grand (réajustement nécessitant un retour sur ce qui a été dit, et réajustement pour trouver une nouvelle $\mathrm{P}$ de base plus adéquate). Rappelons ici au passage que le savoir transposé, disponible pour l'enseignant dans les documents pédagogiques, n'est d'aucune utilité à l'enseignant pour résoudre ce problème spécifique.

\section{Caractéristiques de l'élève chronogène dans l'enseignement grammatical}

La conduite de la chronogenèse revient essentiellement à l'enseignant (Chevallard, 1985/1991). Cependant, certains élèves, dont les interventions, les questions ou les remarques font avancer le savoir ou le présentent autrement, peuvent avoir une fonction chronogène dans la classe (Schubauer-Leoni \& Leutenegger, 2002; Sensevy 1998). Ces élèves ne "conduisent» bien entendu pas l'avancement des savoirs, mais ils y participent, ils y contribuent. En d'autres termes, du point du vue didactique, on ne peut ignorer l'apport de tels élèves si on veut véritablement comprendre le fonctionnement de la chronogenèse ${ }^{18}$.

Il existe à notre avis au moins trois caractéristiques que ces élèves, dits élèves chronogènes (Sensevy, 1998), peuvent présenter relativement au travail grammatical en classe, soit a) la capacité de conciliation, b) l'utilisation des manipulations syntaxiques et c) l'utilisation du métalangage. Ces trois caractéristiques 
sont indépendantes les unes des autres et la présence d'une seule d'entre elles pourrait suffire à rendre un élève chronogène dans un contexte donné. Nous discuterons ici des deux premières ${ }^{19}$.

Précisons ici le fonctionnement de l'argumentation. Nous raisonnons a contrario à partir d'une situation didactique bloquée. Un élève tente, en déployant toutes sortes de moyens (conciliation, utilisation des manipulations syntaxiques et du métalangage), de débloquer la situation, mais ses tentatives échouent. L’examen de ces tentatives dans une situation bloquée nous apparait extrêmement révélateur du fonctionnement des situations didactiques: la force du blocage permet de voir très clairement les mécanismes utilisés par les élèves pour faire avancer une situation didactique. En quoi l'élève est-il chronogène ici? L'élève n'est pas chronogène dans la situation sous étude, qui semble irrémédiablement bloquée, mais il essaie par toutes sortes de moyens de faire avancer le savoir. En supposant que d'autres situations didactiques seraient moins difficiles à débloquer, les caractéristiques dégagées chez l'élève relèvent de la chronogenèse: elles auraient dû faire avancer le savoir dans la classe.

\section{La capacité de conciliation}

Nous appellerons conciliation la résolution par l'élève d'un conflit entre son savoir et le savoir proposé par l'enseignant. Il y a conciliation lorsque l'élève tente de faire coexister de façon cohérente deux connaissances apparemment contradictoires: la conciliation peut être vue comme un cas extrême d'accommodation (au sens de Piaget). La connaissance proposée par l'enseignant n'étant pas assimilable dans les schèmes existants de l'élève, ce dernier doit «accommoder», modifier ses schèmes en conséquence. La conciliation est illustrée plus bas dans l'extrait 4, qui a lieu à un moment où l'enseignant et les élèves ont partiellement reconstruit la $\mathrm{P}$ de base. L'enseignant a écrit la relative au tableau, puis a biffé les groupes au fur et à mesure qu'ils étaient reconstruits par les élèves, comme l'indique la figure suivante.

État du tableau à 17: 28

dont
la beauté égalait la santé

Seul dont n'a pas été biffé: il ne reste plus qu’à insérer dans la $\mathrm{P}$ de base le groupe de mots que dont remplace dans la relative, soit de cette femme, ce qui sera fait dans l'extrait 3 et illustré plus bas. 


\section{Extrait 3}

\begin{tabular}{|l|l|l|l|}
\hline $17: 29$ & E & $\begin{array}{l}\text { Plusieurs élèves } \\
\text { répondent }\end{array}$ & dont \\
\hline & P & & $\begin{array}{l}\text { Dont bon alors ce dont maintenant qu'est-ce qu'on fait avec ça ?... il est } \\
\text { pris... il est mis à la place de quoi ce dont ? }\end{array}$ \\
\hline & E & $\begin{array}{l}\text { Plusieurs élèves } \\
\text { répondent }\end{array}$ & De la femme \\
\hline $17: 36$ & $\mathrm{P}$ & & De la femme $\uparrow$ \\
\hline $17: 37$ & $\mathrm{P}$ & & $\begin{array}{l}\text { Alors comment est-ce qu'on peut compléter cette phrase (pointe la } \\
\text { phrase du bas au tableau) en rajoutant la femme }\end{array}$ \\
\hline & E & & La beauté égale (?) la santé de la femme $\uparrow$ \\
\hline & $\mathrm{P}$ & & De la femme $\uparrow$ \\
\hline
\end{tabular}

\section{État du tableau à 17:52}

\section{labeate egataté santé \\ la beauté égalait la santé de la ferme}

Les deux interventions qui suivent ont été faites par un même élève. Elles ont lieu au moment même où l'enseignant place le SP de la femme à la fin de la phrase comme complément de santé et ne seront pas relevées par ce dernier.

\section{Extrait 4}

\begin{tabular}{|l|l|l|}
\hline $17: 46$ & E1 & Bon alors avec le dont on va le mettre à la fin \\
\hline $17: 50$ & E1 & On va le mettre à la fin fallait (le dire ? l'écrire ?) au début \\
\hline
\end{tabular}

La première intervention témoigne de la tentative de l'élève de concilier deux savoirs: «bon alors avec le dont on va le mettre à la fin». Elle peut s'interpréter comme suit: on va placer le SP à la fin de la phrase, même s'il va en fait ailleurs (au début, après beauté). L'élève tente de formuler une règle qui lui permette de retourner à la $\mathrm{P}$ de base lorsque dont est employé, règle qui soit conforme à la fois à son intuition (le SP va au début, ou peut aller au début - il s'agit du premier savoir) et à ce que l'enseignant propose (on le place à la fin - il s'agit du deuxième savoir, qui entre en contradiction avec le premier). En d'autres termes, l'élève aurait choisi une autre place pour le SP (au début) mais tente d'intégrer le savoir proposé par l'enseignant et de le concilier avec le sien: il énonce donc une condition pour mettre le groupe «à la fin», à savoir que le pronom relatif soit dont («avec le dont»).

Lélève verbalise son processus d'accommodation, de conciliation, et cela met au jour le raisonnement qui lui permet d'intégrer la connaissance nouvelle. Cette capacité à verbaliser son processus de conciliation fait à notre avis partie des caractéristiques d'un élève chronogène. En effet, le fait d'énoncer une condition d'application d'un savoir, comme l'élève le fait, précise le contexte dans lequel ce 
dernier est pertinent: il n'y a pas à douter que si le professeur ne précise pas les conditions de validité d'une connaissance, un élève qui le ferait serait chronogène.

\section{L'utilisation des manipulations syntaxiques}

Une des caractéristiques d'un élève chronogène dans le contexte de l'enseignement/apprentissage de la grammaire devrait à notre avis être la capacité d'utiliser les manipulations syntaxiques pour faire comprendre son raisonnement. L'extrait 5 illustre une utilisation magistrale par l'élève de ces manipulations, et il nous semble clair que, en l'absence de blocage de la situation didactique, cette intervention aurait contribué à l'avancement du savoir dans le temps.

Dans l'extrait 5, l'élève essaie de démontrer qu'un SP provenant du sujet ne peut être reconstruit à la fin de la phrase, à l'intérieur du complément direct (CD). Il choisit donc une autre phrase (donnée en 21) dans le corpus fourni par l'enseignant et effectue la démonstration à partir de cette phrase.

(21) Un étranger dont [les yeux ] projetaient des flammes

\section{Extrait 5}

\begin{tabular}{|c|c|c|}
\hline $18: 59$ & E1 & $\begin{array}{l}\text { Ça veut dire qu'à dont on peut mettre le...enfin le sujet, heu... on peut mettre le sujet }{ }^{19} \\
\text { n'importe où dans la phrase }\end{array}$ \\
\hline & P & On peut pas mettre le sujet n'importe où dans la phrase \\
\hline 19:10 & E1 & Non.. mais heu... mais celui qu'on reprend par le dont \\
\hline 19:12 & $\mathrm{P}$ & On peut pas le mettre n'importe où, non \\
\hline \multicolumn{3}{|l|}{$(\ldots)$} \\
\hline & E1 & Mettons pour la cinquième \\
\hline & P & oui \\
\hline \multirow[t]{6}{*}{$19: 35$} & E1 & $\begin{array}{l}\text { On peut pas dire les yeux projetaient des flammes de l'étranger } \\
\text { On est obligé de le mettre après... après heu les yeux XXX les yeux de l'étranger projetaient } \\
\text { des flammes }\end{array}$ \\
\hline & P & les yeux de l'étranger... donc on peut pas le mettre n'importe où $\uparrow$ (sourire) \\
\hline & E1 & $\begin{array}{l}\text { Ben si, ben oui, ben non on peut pas le mettre n'importe où mais on peut le mettre dans } \\
\text { la phrase heu... n'importe où dans la phrase... }\end{array}$ \\
\hline & $\mathrm{P}$ & On est obligé de le mettre à l'endroit où ça correspond au sens \\
\hline & E1 & Mais on peut le mettre dans la phrase \\
\hline & $\mathrm{P}$ & Voilà $\uparrow$ d'accord on peut le mettre dans la phrase, ailleurs dans la phrase. \\
\hline
\end{tabular}

Soulignons la qualité du travail de l'élève dans cette comparaison. D'abord, il choisit un exemple pertinent pour effectuer la comparaison. En effet, dans un étranger dont les yeux projetaient des flammes, tout comme dans une femme dont la beauté égalait la santé, dont est extrait du sujet et il y a un CD. La P de base correspondante est donnée en (22). 


\section{(22) Les yeux de l'étranger projetaient des flammes}

Ensuite, l'élève identifie correctement le SP que dont remplace, soit de l'étranger. Il reconstruit la $\mathrm{P}$ de base en plaçant de l'étranger dans le CD, comme l'enseignant l'a fait pour la phrase à l'étude. L'élève souligne l'agrammaticalité de sa reconstruction («on ne peut pas dire...»). Sa phrase reconstruite, donnée en (23a) est strictement parallèle à $\mathrm{P}_{\mathrm{I}}$, rappelée en $(23 \mathrm{~b})$.

(23) a. *Les yeux projetaient des flammes de l'étranger.

b. La beauté égalait la santé de la femme.

Enfin, l'élève compare (23a) avec la reconstruction du SP dans le sujet (en 24a) et souligne la grammaticalité du résultat («on est obligé de le mettre après (...)les yeux»). Notons que cette reconstruction est tout à fait parallèle à celle qu'il a essayé de proposer pour la phrase (10), rappelée en (24b).

(24) a. Les yeux de l'étranger projetaient des flammes

b. La beauté de la femme égalait la santé

Il nous semble indéniable que la maitrise des manipulations syntaxiques, dans une situation didactique non bloquée, ne pourrait que contribuer à faire avancer le savoir au fil du temps.

\section{Conclusion}

À travers l'étude d'interactions didactiques portant sur le travail grammatical en classe de français, nous avons proposé qu'il existe certaines conditions topogénétiques à la conduite de la chronogenèse, et en avons identifié deux qui nous semblent particulièrement saillantes, notamment pour le travail grammatical, soit l'anticipation des hypothèses alternatives des élèves et la clarté des représentations de l'enseignant relativement au savoir à enseigner. La position de l'enseignant par rapport au savoir à enseigner, le fait qu'il sache «autrement» (Chevallard 1985/1991), est à notre avis ce qui lui permet de prévoir les points de bifurcation possible dans le raisonnement des élèves. Les représentations de l'enseignant relativement au savoir à enseigner, en particulier ses représentations implicites, influencent sa conduite de la chronogenèse. Pour l'enseignement grammatical, nous avons vu qu'une contrainte implicite sur la reconstruction de la P de base (le principe d'unicité), à notre connaissance jamais discutée dans les travaux de transposition didactique, pouvait influencer le cours de la chronogenèse.

Les extraits choisis nous ont également permis d'identifier certaines caractéristiques d'un élève chronogène pour l'enseignement grammatical, soit la capacité de concilier différents savoirs et de verbaliser cette conciliation, de même que la 
maitrise des manipulations syntaxiques. Il n'est pas nécessaire pour un élève de cumuler ces caractéristiques pour être chronogène.

L'observation et l'analyse des interactions didactiques en classe de français constitue à notre avis un champ de recherche prometteur pour enrichir la théorisation de la transposition didactique et des situations didactiques de façon générale, et également pour comprendre la particularité de certains mécanismes didactiques spécifiques à l'enseignement grammatical, notamment dans une perspective de formation initiale et continue des enseignants.

\section{Notes}

1 Cet article a été amorcé lors d'un stage postdoctoral à l'université de Genève au printemps 2004. Je remercie Joaquim Dolz et Bernard Schneuwly de m'avoir si gentiment donné accès à leur corpus et d'avoir eu avec moi de nombreuses discussions au sujet de cet article. Je remercie également Sandra Canelas-Trevisi pour nos discussions.

2 Pour une discussion de la transposition didactique externe dans la discipline français, voir le numéro spécial de Pratiques 1998 et Schneuwly (2005). En plus des savoirs savants des disciplines contributoires, on considère généralement que les pratiques sociales de référence doivent être prises en compte dans la transposition. La transposition externe des savoirs savants sur la subordonnée relative est brièvement discutée plus loin.

3 Les données ont été recueillies dans le cadre du projet La construction de l'objet enseigné en classe de français. Analyse du travail enseignant en grammaire et en production écrite, soutenu par le Fonds national suisse de la recherche scientifique (1214-068110) et dirigé par Joaquim Dolz et Bernard Schneuwly.

4 Nous utilisons dans cet article la terminologie linguistique et employons le terme syntagme; dans le matériel didactique, le terme groupe est généralement utilisé pour désigner la même réalité.

5 L'analyse de la grammaire générative differe de celle exposée dans les grammaires pédagogiques. En effet, dans l'analyse généralement acceptée en grammaire générative, que n’est pas un pronom mais bien un complémenteur (subordonnant), généré directement en tête de phrase et introduisant la subordonnée. Un opérateur vide $(O p)$ occupe la position de base du syntagme et se déplace devant le complémenteur, laissant une trace $(t)$ dans sa position initiale, ce qui assure qu'aucun autre élément ne pourra l'occuper. La dérivation de la phrase (1) dans ce cadre est donnée en (1').

$\left(1^{\prime}\right)$ a. Le phénomène [ que Jean a expliqué $O p_{i}$ ] est passionnant.

b. Le phénomène $\left[O p_{i}\right.$ que Jean a expliqué $\left.t_{i}\right]$ est passionnant.

Le lecteur intéressé pourra consulter avec profit Tellier et Valois (2006, pp. 26-31) et Tellier (2003, pp. 140-147) pour une présentation claire des arguments fondant cette analyse.

6 Pour une discussion des syntagmes auxquels dont se substitue en français, voir Boivin (1999, 2005), de même que Boivin, Pinsonneault et Philippe (2003), Riegel, Pellat et Rioul (2004, pp. 209 et 482).

7 En grammaire générative, cette contrainte est exprimée par le principe $\mathrm{C}$ de la théorie du liage (Binding Theory). La théorie du liage rend compte de la distribution des expressions référentielles, des pronoms et des anaphores dans la phrase (cf. Chomsky, 1981, 1986). Pour une introduction en français à la théorie du liage, voir Pollock (1997).

8 Lanalyse syntaxique indique que dont provient du sujet et non du complément, comme le montre l'asymétrie dans la grammaticalité des phrases suivantes.

Il est possible d'extraire dont à partir du sujet la beauté de cette femme, comme en (ii).

(i) [La beauté de cette femme] égalait la santé de Marie

(ii) La femme dont [la beauté_] égalait la santé de Marie 
La phrase (iv) montre l'agrammaticalité de l'extraction de dont à partir de l'objet la santé de cette femme.

(iii) La beauté de Marie égalait [la santé de cette femme]

(iv) ${ }^{*}$ La femme dont la beauté de Marie égalait [la santé _ ]

Bien entendu, cette observation est vraie dans le contexte où le sujet est de la forme $\mathrm{N}$ de $\mathrm{N}$. Autrement, dont peut être extrait d'un objet (cf. La femme dont j’ai rencontré la sour hier). Pour une analyse syntaxique récente des conditions d'extraction de dont, et en particulier des asymétries sujet/objet qu'on y observe, voir Boivin (1999, 2005).

9 En voici d'autres exemples, tirés de la thèse de Tellier (1991).

(i) ...un vieux vigogne dont la plume_ cache les trous_

Edmond Rostand, Cyrano de Bergerac

(ii) Cette sombre histoire, dont les images_ s saccordent parfaitement au rythme__ et au ton

René Homier-Roy, Châtelaine, décembre 1987

(iii) Noam Chomsky, dont l'apport__à la linguistique est moins sujet à caution que les prises de position politiques

Philippe Videlier, Le Monde diplomatique, novembre 1990

10 L'exposé est fait en fonction de la description transposée. Rappelons que dans l'analyse standard de la grammaire générative, le "pronom relatif» est directement généré comme complémenteur (cf. note iv); la position d'origine du pronom relatif dans le texte correspond donc à la position d'origine de l'opérateur $O p$.

11 Au fil de la leçon, il y aura un changement de déterminant dans le SP, qui passera de de cette femme à de la femme. Ce fait n'affecte pas notre discussion; nous utiliserons les phrases telles qu'elles ont été produites en classe.

12 Un évaluateur a fait remarquer que l'expression conduite de la chronogenèse tend à réifier un concept construit par les didacticiens, comme si l'enseignant pouvait exercer un contrôle sur cet objet construit. Il faut à mon avis comprendre cette expression de Chevallard comme un raccourci indiquant simplement que la chronogenèse est fortement déterminée par les actions de l'enseignant. En d'autres termes, la description et la compréhension de la chronogenèse exige de se pencher sur les actions de l'enseignant. Sur le rôle de l'élève dans la chronogenèse, voir la sixième partie de l'article.

13 Italiques, guillemets et orthographe issus du texte original.

14 Croché est un participe passé adjectival ayant le sens (figuré) de accroché. (Dictionnaire suisse roman).

15 En italiques dans le texte.

15 Cette prise en compte est à lier à la capacité d'anticipation (Chevallard 1985/91, p. 72) de l'enseignant.

17 Comme nous le verrons, la suggestion de l'élève sera rejetée parce qu'elle impliquerait de rajouter un mot à la phrase: «La beauté de cette femme égalait SA santé». Nous reviendrons sur cette phrase et sur ce qu'elle implique comme règles implicites de reconstruction de la $\mathrm{P}$ de base dans la cinquième partie de l'article.

18 À propos de la participation des élèves à l'avancement des savoirs, voir également CohenAzria et al. (2007), sous la rubrique chronogenèse.

19 Le contraste entre l'utilisation du métalangage (savoir déclaratif) et l'utilisation des manipulations syntaxiques (savoir procédural) chez un même élève est présenté et discuté dans Boivin (à paraître).

19 Avec le terme «le sujet» l'élève semble référer au groupe prépositionnel remplacé par dont, ici de la femme. Pour une discussion du métalangage dans cet extrait, voir Boivin (à paraître). 


\section{Bibliographie}

Boivin, M.-C. (1999). Split Noun Phrases and the Theory of Case. Thèse de doctorat, Massachusetts Institute of Technology, Cambridge: MIT Working Papers in Linguistics.

Boivin, M.-C. (2005). Case Theory, DP Movement, and Interpretation: A New Approach to the Distribution of French Clitic en. Natural Language and Linguistic Theory, 23, 543-593.

Boivin, M.-C. (à paraître). Savoirs déclaratifs et procéduraux d'un élève chronogène dans le travail grammatical. In S. Canelas-Trevisi (Ed.), Actes du colloque Langage, objets enseignés et travail enseignant en didactique du français, Université de Grenoble, 17 et 18 mai 2005.

Boivin, M.-C., Pinsonneault, R. \& Philippe, M.-E. (2003). Bien écrire: la grammaire revue au fil des textes littéraires. Laval: Beauchemin.

Brousseau, G. (1998). Théorie des situations didactiques. Textes rassemblés et préparés par Nicolas Balacheff, Martin Cooper, Rosamund Sutherland, Virginia Warfield. Grenoble: La Pensée sauvage.

Canelas-Trevisi, S. (1998). Manipuler des phrases et en parler: action et (méta)langage en classe de français. In J. Dolz \& J.-C. Meyer (Ed.), Activités métalangagières et enseignement du français. Actes des journées d'étude en didactique du français de Cartigny, 28 février au 1er mars 1997 (pp. 70-89). Berne: Peter Lang.

Canelas-Trevisi, S., Moro, C., Schneuwly, B. \& Thévenaz, T. (1999). L'objet enseigné: vers une méthodologie plurielle d'analyse des pratiques d'enseignement en classe. Repères, 20, 143-162.

Chartrand, S.-G. (Ed.). (1996). Pour un nouvel enseignement de la grammaire, 2e édition. Montréal: Éditions Logiques.

Chevallard, Y. (1985/1991). La transposition didactique. Du savoir savant au savoir enseigné. Grenoble: La pensée sauvage.

Chomsky, N. (1981). Lectures on Government and Binding. Dordrecht: Foris.

Chomsky, N. (1986). Knowledge of Language. Its Nature, Origin, and Use. New York: Praeger.

Chomsky, N. (1995). The Minimalist Program. Cambridge: MIT Press.

Cohen-Azria, C., Daunay, B., Delcambre-Delville, I., Lahanier-Reuter, D. \& Reuter, Y. (2007). Dictionnaire des concepts fondamentaux des didactiques. Bruxelles: De Boeck.

DeKeyser, R. (2003). Implicit and Explicit Learning. In C. Doughty \& M. Long (Ed.), Handbook of Second Language Acquisition (p. 313-348). Oxford: Blackwell.

Fetzer, A. (2004). Recontextualizing Context. Grammaticality Meets Appropriateness. Amsterdam: John Benjamins.

Genevay, E. (1994). Ouvrir la grammaire. Lausanne, Montréal: LEP et La Chenelière.

Gobbe, R. (1980). Pour appliquer la grammaire nouvelle, 2e édition. Bruxelles: De Boeck.

Kak, M. B. (1992). Grammars and Grammaticality. Amsterdam: John Benjamins.

Leutenegger, F. \& Schubauer-Leoni, M.-L. (2002). Les élèves et leur rapport au contrat didactique: une perspective de didactique comparée. Les Dossiers des Sciences de l'Education, No 8 (pp. 73-86), Toulouse: Presses universitaires du Mirail.

Nadeau, M. \& Fisher, C. (2006). La grammaire nouvelle. La comprendre et l'enseigner. Montréal: Gaëtan Morin Éditeur/Chenelière Éducation.

Pollock, J.-Y. (1997). Langage et cognition. Introduction au programme minimaliste de la grammaire générative. Paris: PUF.

Riegel, M., Pellat, J.-C. \& Rioul, R. (1994/2004). Grammaire méthodique du français. Paris: PUF.

Sensevy, G. (1998). Institutions didactiques. Etude et autonomie à l'école élémentaire. Paris: PUF.

Schneuwly, B. (2005). De l'utilité de la transposition didactique. In J.-L. Chiss, J. David \& Y. Reuter (Ed.), Didactique du français: fondements d'une discipline (pp. 47-59). Bruxelles: De Boeck.

Schubauer-Leoni, M.-L. \& Dolz, J. (2004). Comprendre l'action et l'ingéniosité didactique de l'enseignant: une composante essentielle de la transformation de l'Ecole. In J.-P. Bronckart \& M. Gather Thurler (Ed.), Transformer l'école (pp. 147-168). Bruxelles: De Boeck. 
Schubauer-Leoni, M.-L. \& Leutenegger, F. (2002). Expliquer et comprendre dans une approche clinique/expérimentale du didactique ordinaire. In F. Leutenegger \& M. Saada-Robert (Ed.), Expliquer et comprendre en sciences de l'éducation (pp. 227-251). Raisons Educatives. Bruxelles: De Boeck

Tardif, J. (1992). Pour un enseignement stratégique. Montréal: Les Éditions Logiques.

Vargas, C. (2004). La création des savoirs à enseigner en grammaire: de la recomposition à la reconfiguration. In C. Vargas (Ed.), Langue et études de la langue: approches linguistiques et didactiques, Actes du colloque international de Marseille (pp. 35-47). Aix-en-Provence: Presses de l'Université de Provence.

Tellier, C. (1991). Licensing Theory and French Parasitic Gaps. Dordrecht: Kluwer.

Tellier, C. (2001). On Some Distinctive Properties of Parasitic Gaps in French. In P. W. Culicover \& P. M. Postal (Eds), Parasitic Gaps (pp. 341-367). Cambridge: Massachusetts Institute of Technology.

Tellier, C. (2003). Eléments de syntaxe du français. Méthodes d'analyse en grammaire générative. $2 e$ édition. Montréal: Gaetan Morin éditeur/Chenelière Education.

Tellier, C. \& Valois, D. (2006). Constructions méconnues du français. Montréal: Les presses de l'Université de Montréal.

Wirthner, M. \& Schneuwly, B. (2004). Variabilité et contrainte dans la construction des significations d'un objet d'enseignement. L'effet d'un outil pour enseigner le résumé d'un texte informative. In C. Moro \& R. Rickenmann (Ed.), Situation éducative et significations (pp. 107-133). Bruxelles: De Boeck.

Mots clés: didactique de la grammaire, connaissances implicites, interactions didactiques, transposition didactique interne, enseignement secondaire

\section{Didaktische Situationen im Grammatikunterricht: Topogenetische und chronogenetische Aspekte}

\section{Abstract}

Der Beitrag basiert auf Untersuchungen zu didaktischen Interaktionen im Französischunterricht (Thema: Satzmodelle). Die Ergebnisse weisen auf zwei Bereiche im Verlauf didaktischer Situationen hin. Es wird gezeigt, dass topogenetische Bedingungen (Topogenese, verstanden als Entwicklungsraum von LehrerInnen wie auch von SchülerInnen in Auseinandersetzung mit dem Wissen) für den Aufbau und die Entwicklung von Wissen in einer Klasse (Chronogenese, verstanden als gesteuerter Prozess des Wissensaufbaus in einer Klasse) bestehen: Entscheidend sind auf Seiten des Lehrers/der Lehrerin die Antizipation der Generierung von Alternativhypothesen durch die SchülerInnen und die Eindeutigkeit des Unterrichts in Relation zum Unterrichtsgegenstand. Zwei zentrale Fähigkeiten der SchülerInnen werden anhand einer Grammatiklektion vorgestellt und illustriert: Der bewusste Umgang mit, wie auch die Nutzung syntaktischer Veränderungen. Dabei wird auch die Rolle des impliziten grammatikalischen Wissens der Schüler diskutiert.

Schlagworte: Didaktik der Grammatik, implizites Wissen, didaktische Interaktionen, interne didaktische Umsetzung, Sekundarstufe 


\title{
Situazioni didattiche e insegnamento della grammatica: aspetti topo-e cronogenetici
}

\section{Riassunto}

Questo articolo si fonda sull'analisi delle interazioni didattiche attinenti alla frase principale e alla subordinata nelle classi di francese. Per l'evolversi delle situazioni didattiche si fanno due supposizioni: esistono i) condizioni topogenetiche che condizionano ii) l'evoluzione del sapere in classe (cronogenesi). Più precisamente: l'anticipazione delle ipotesi alternative degli allievi e la chiarezza delle rappresentazioni dell'insegnante circa i saperi da insegnare sono cruciali. Vengono poi illustrate due caratteristiche di un allievo cronogenetico nel contesto di una lezione di grammatica: la capacità di conciliare e l'utilizzazione delle manipolazioni sintattiche. Infine si discute il ruolo delle conoscenze grammaticali implicite (competenza linguistica) degli allievi.

Parole chiave: Didattica della grammatica, conoscenze implicite, interazioni didattiche, trasposizioni didattiche interne, livello secondario

\section{Didactic situations in the teaching of grammar: Topogenetic and chronogenetic aspects}

\begin{abstract}
This paper relies on the analysis of didactic interactions on the basic sentence model and on relative clauses in a French classroom, and makes two claims regarding the unfolding of didactic situations. It is proposed that there are topogenetic conditions on the advancement of knowledge in class as time goes by (chronogenesis), more specifically that the anticipation of the students' alternative hypotheses as well as a clear representation of the knowledge to be taught are crucial. Two characteristics of a chronogenetic student are proposed and illustrated in a grammar lesson: a conciliation capacity, and the use of syntactic manipulations. The role of implicit grammatical knowledge (linguistic competence) is discussed.
\end{abstract}

Keywords: grammar instruction, implicit knowledge, classroom interactions, internal didactic transposition, secondary education 
